\title{
THE CLASSIFICATION OF RATIONAL APPROXIMATIONS
}

\author{
By P. J. Heawood.
}

[Received September 20th, 1920.-Read November 11th, 1920.]

1. In the Proceedings of the London Mathematical Society for January 14th, 1919,* various interesting questions are raised by Mr. J. H. Grace with respect to the rational approximations $x / y$, to a given (incommensurable) quantity $\theta$, which satisfy the condition $|x / y-\theta|<1 / k y^{2}$, where $k$ is a given number, $x / y$ being a fraction in its lowest terms. Among other things he refers to a paper of Markoff's, $\dagger$ on which he bases the statement that, if $k \leqslant 3$, there will be an infinite number of such approximations except only in certain cases where $\theta$ is a quadratic surd. As a matter of fact Markoff's results are not precisely on the same footing as those required for our purpose, as will appear in the sequel, and the true condition above is $k<3$, not $k \leqslant 3$; but the question at once arises whether, if $k$ is only just greater than 3 , the reverse is the case; i.e. whether for such values of $k$ there can be transcendent numbers $\theta$, for which there are only a finite number of approximations such that $|x / y-\theta|<1 / k y$. $\quad$ Mr. Grace proceeds to show that such numbers can be constructed for $k=3.0322$ (or any greater value). The whole theory of such approximations depends on the fact that if $|x / y-\theta|<1 / k y^{2}$ for such a $k$, or indeed for any value of $k \geqslant 2, x / y$ must be a convergent $p_{n} / q_{16}$ to the continued fraction for $\theta$. Then, if

$$
\theta=\left[a_{0}\right]+\frac{1}{a_{1}}+\frac{1}{a_{2}}+\cdots \quad\left[a_{0} \text { perhaps zero }\right],
$$

we have

$$
|x / y-\theta|=1 / \lambda y^{2} \text {, }
$$

where

$$
\lambda=\left(a_{n+1} ; a_{n+2}, a_{n+3}, \ldots\right)+\left(a_{n}, a_{n-1}, \ldots, a_{1}\right) ;
$$

since

$$
\theta=\left(x p_{n}+p_{n-1}\right) /\left(a q_{n}+q_{n-1}\right)
$$


where

$$
\begin{gathered}
a=\left(a_{n+1} ; a_{n+2}, a_{n+3}, \ldots\right), \\
q_{n-1} / q_{n}=\left(a_{n}, a_{n-1}, \ldots, a_{1}\right) ;
\end{gathered}
$$

while

using the abbreviated notation for

$$
a_{n+1}+\frac{1}{a_{n+2}}+\cdots \text { and } \frac{1}{a_{n}}+\frac{1}{a_{n-1}}+\cdots
$$

Supposing then (to take Mr. Grace's application), that in the continued fraction for $\theta$ the denominators consist of cycles of $m$ 1's and $m 2$ 's, each used any number of times in succession, the greatest values of $\lambda$ will be numbers of the form $(2 ; 2,2, \ldots)+(1,1,1, \ldots)$, which by taking $m$ large enough can be brought as near as we please to

$$
\sqrt{ } 2+1+\frac{1}{2}(\sqrt{ } 5-1)=3 \cdot 032 \ldots .
$$

If, then, $k$ exceeds this limit, numbers $\theta$ can be thus constructed which have no approximations $x / y$ such that $|x / y-\theta|<1 / k y^{2}$. But, as the set of such numbers is unenumerable, it must include non-algebraic numbers.

The author suggests that it ought to be possible (if 3 is really the limiting value) so to choose our cycles that the critical value of $\lambda$ is brought down to 3, but does not see his way to do so. If, however, we take, instead of cycles of $m 1$ 's and $m 2$ 's, cycles consisting the one of $m 1$ 's, the other of two 2 's followed by $m-21$ 's, used precisely as above, this is accomplished. For the maximum value of $\lambda$ will now be

$$
(2 ; 2,1,1, \ldots)+(1,1,1, \ldots),
$$

or, which is the same thing,

$$
(2,1,1, \ldots)+(2 ; 1,1,1, \ldots) ;
$$

which tends to $\frac{1}{2}(3+\sqrt{ } 5)+\frac{1}{2}(3-\sqrt{ } 5)$, i.e. 3 , as $m$ is indefinitely increased. If, then, $k$ is ever so slightly greater than 3 , we can construct transcendental numbers $\theta$ for which there are no approximations such that $|x / y-\theta|<1 / k y^{2}$.

2. The main question, however, is as to the structure of numbers $\theta$ for which there are (at most) only a finite number of approximations $x / y$ such that $|x / y-\theta|<1 / k y^{2}$, when $k \leqslant 3$; i.e. where, at least beyond a certain point, when $\theta$ is reduced to a continued fraction, every $\lambda<3$ $\left[\lambda=3\right.$, i.e. $|x| y-\theta \mid=1 / 3 y^{2}$, is impossible with $\theta$ incommensurable] Towards this, Markoff's assistance is somewhat incidental. His main problem is that of the minima of quadratic forms of positive determinant $D$; and, imagining from Mr. Grace's paper that Markoff's whole theory 
was mixed up with that of these forms, I made an independent analysis. Having now read Markoff's article, I find that his continued fraction analysis, though merely subsidiary to his work on the minima of quadratic forms, is really independent of it, and largely on parallel lines with mine. There are, however, many differences in detail and a certain want of symmetry in his classification of types, which obscures some points of special interest. His notation, too, is rather cumbrous and his logical analysis is not very complete. After showing that certain alternatives must be rejected and that certain others are possible, he is content to conclude "De toutes ces considérations il suit, que la suite cherchée présentera l'une des formes suivantes....", without showing clearly how the result is arrived at. I therefore venture to give my analysis exactly as I worked it out independently, referring in footnotes to any important divergences. Moreover Markoff's work is not exactly on all fours with that required for our purpose. The quantities which he has to examine, as to their being continually equal to or less than 3 , are of the same form as $\lambda$ above, but with the important difference that his $a$ 's extend without limit in both directions, so that neither of the two fractions

$$
\left(a_{n+1} ; a_{n+2}, a_{n+3}, \ldots\right), \quad\left(a_{n}, a_{n-1}, \ldots\right),
$$

whose sum is $\lambda$, terminates. One result is that it is possible for his $\lambda^{*}$ to be equal to 3 , in certain cases. This does not affect the general course of the analysis. It has, however, to be borne in mind in the final conclusions.

3. Proceeding then to consider the classes of continued fractions for which, beyond a certain point, no $\lambda$ (as defined above) is greater than 3, we can see at the outset that they will be of very restricted types. To begin with, the expression for $\lambda$ shows that, from the point in question, no denominators can be as great as 3 ; they must consist of the digits 1 and 2 only. Again, there cannot be an isolated 2 in the midst of 1 's, since $(2 ; 1, \ldots)+(1, \ldots)>3$, whatever digits follow; nor can there be an isolated 1 in the midst of 2 's, since $(2 ; 1,2, \ldots)+(2, \ldots)>3$, whatever digits follow; thus the 2's must occur in groups of 2 or more and likewise the 1's. On the other hand, the "points of danger" will occur: only when there is a transition from 1's to 2's or from 2's to 1's. If $a_{n+1}$ be a 1 in the midst of 1's, $\dagger$ or a 2 in the midst of 2's, the corresponding $\lambda$ cannot exceed 3 , since $(2 ; 2, \ldots)+(2, \ldots)<3 ;(1 ; 1, \ldots)+(1, \ldots)<3$,

* 2/L in his notation: then the least value of $L \sqrt{ } D$ is the minimum of the form.

$\dagger$ Or if $a_{n+1}=1$, in any case. 
whatever digits follow those specified. One case therefore which will do is where (after some point) the digits are all 2's or all 1's. Supposing, however, that both 2's and 1's occur throughout, we have only to examine the values of $\lambda$ where $a_{n+1}$ is the first or last of a succession of 2's.

Where the digits consist merely of 1's and 2's, we may adopt a still further abridged notation for the continued fractions involved. Let $[p|q| r \ldots]$ stand for

$$
\frac{1}{1}+\frac{1}{1}+\cdots+\frac{1}{2}+\frac{1}{2}+\cdots
$$

where there are $p$ 1's, followed by $q 2$ 's, followed by $r$ 1's, etc.; and $\{p|q| r \ldots\}$ in like manner for

$$
\frac{1}{2}+\frac{1}{2}+\cdots+\frac{1}{1}+\cdots
$$

where there are first $p 2$ 's, then $q 1$ 's, then $r$ 2's, etc. That the $\lambda$ corresponding to the first of a set of $r 2$ 's may be less than 3 , we have, say, ${ }^{*}$

$$
\left(2+\frac{1}{2}+\frac{1}{x}\right)+\left(\frac{1}{1}+\frac{1}{1}+\frac{1}{y}\right)<3,
$$

where $x$ and $y$ stand for the aggregates which follow;

i.e.

$$
\frac{1}{2}+\frac{1}{x}+\frac{y+1}{2 y+1}<1
$$

i.e.

$$
\frac{1}{2}+\frac{1}{x}<\frac{y}{2 y+1}, \text { i.e. }<\frac{1}{2}+\frac{1}{y}
$$

i.e.

$$
\frac{1}{x}>\frac{1}{y} \text {. }
$$

Using the abridged notation just explained, if we suppose the $r$ 2's followed by $s 1$ 's, then $t 2$ 's, etc., and preceded by $q 1$ 's, then $p 2$ 's, etc., the condition is

$$
\{r-2|s| t \ldots\}>[q-2|p| o \ldots],
$$

the two sides of the inequality being the values of $1 / x$ and $1 / y$ respectively. Since an interchange of the digits 1 and 2 throughout will make the larger fraction the smaller, this is precisely the same thing as:-

$$
\{q-2|p| o \ldots\}>[r-2|s| t \ldots] \text {. }
$$

Dealing in like manner with the $\lambda$ corresponding to the last of the $r$ 's,

* We have already seen that both 1's and 2's occur in groups of two or more. 
we have a condition which may indifferently be written

or

$$
\begin{aligned}
& \{r-2|q| p \ldots\}>[s-2|t| u \ldots], \\
& \{s-2|t| u \ldots\}>[r-2|q| p \ldots] .
\end{aligned}
$$

If two such conditions are satisfied for each group of 2's, ${ }^{*}$ it will secure that we have a fraction for which every $\lambda$ is less than 3 . Com paring them in the forms numbered (1), (2) above, it will be seen that (2) is exactly the same relatively to $r, s, \ldots$ that (1) is relatively to $q, r, \ldots$. If, then, the numbers $\ldots o, p, q, r, s, t, \ldots$ are those of the 2 's and 1 's alternately, it does not really matter which of the alternate sets are 2's and which are 1's, so far as the conditions for $\lambda<3$ are concerned. We may take (2) as the typical form of condition, which must be satisfied for each "transition," whether it really be from 2's to 1's or from 1's to 2's. $\dagger$

4. For the typical condition

$$
\{s-2|t| u \ldots\}>[r-2|q| p \ldots]
$$

to be possible we must have either $r=2$ or $s=2$ (or both), since a fraction beginning $\frac{1}{2}+\ldots$ cannot be greater than one beginning $\frac{1}{1}+\ldots$; i.e. of two consecutive sets of digits, one at least must be a doublet, whether of 1's or of 2's. [If both $r=2$ and $s=2$, the condition becomes $[t \mid u \ldots]>\{q \mid p \ldots\}$ which is necessarily satisfied.] In any case we have

$$
\text { either } r=2 \text { and }\{s-2|t| u \ldots\}>\{q|p| 0 \ldots\} \text {, }
$$

which is equivalent to

$$
[q|p| o \ldots]>[s-2|t| u \ldots]
$$

$$
\text { or else } s=2 \text { and }
$$

$$
[t \mid u \ldots]>[r-2|q| p \ldots] \text {. }
$$

These are the conditions for the $r$-s "transition." For the $q-r$ transition there will be similar conditions involving either $q=2$ or $r=2$. Suppose $r=2$. Then the conditions for the $q-r$ and $r$-s transitions,

* Markoff's analysis is complicated by the fact that, though be begins with a transition from 2's to 1's, he does not confine himself to such crucial points nor treat them comprehensively. [He uses throughout the fullest expressions for the continued fractions involved.]

† This "duality" is not observed by Markoff, and this affects the symmetry of his work. Further his final conclusions do not show explicitly the correspondence throughout of cases. in which 1's and 2's are interchanged. 
as above, reduce to

and

$$
[s|t| u \ldots]>[q-2|p| o \ldots],
$$

We have to see how these can be simultaneously satisfied. Of the alternatives $q-2<s, s-2<q$, one (or both) must necessarily be true for any pair of numbers $q, s$. Suppose $q-2<s$. Then in the fractions compared in (i), the transition from the 1-digits (with which each begins) to the 2-digits occurs earlier on the right hand than on the left. For the fraction on the right to be smaller, the first 2-digit of the $p$-set, answering there to 1 in the other, must come in an odd place; for

$$
\frac{1}{1}+\frac{1}{2}+\cdots>\frac{1}{1}+\frac{1}{1}+\cdots
$$

but

$$
\frac{1}{1}+\frac{1}{1}+\frac{1}{2}+\cdots<\frac{1}{1}+\frac{1}{1}+\frac{1}{1}+\cdots,
$$

whatever digits follow, and so on. Thus $q-2$ must be even and so $q$ must be even. Then (i) is satisfied; but for (ii) to hold, $s-2$ cannot be greater than $q$, or ( $q$ being even) the left-hand fraction in (ii) would be the smaller; but either $s-2<q$ and $s$ even, which with the preceding entails $s=q$ and both even; or else $s-2=q$, and then ( $q$ being even) (ii) reduces to

$$
\{p \mid 0 \ldots\}>\{t \mid u \ldots\},
$$

which must also be satisfied. Similarly for the case $q-2=s$.

By considering a typical transition we thus reach the conclusion that for the requisite conditions to be satisfied throughout:-

(1) All the numbers ...,o, $p, q, r, s, t, u, \ldots$ must be even;

(2) Of any two consecutive numbers one at least must $=2$;

(3) If $r=2$, we have further:-

$$
\begin{array}{ll}
\text { either }(\text { (a) } q=s ; \\
\text { or } \quad \text { (b) } s=q+2, \quad\{p \mid o \ldots\}>\{t \mid u \ldots\} ; \\
\text { or } \quad \text { (c) } q=s+2, \quad\{t \mid u \ldots\}>\{p \mid o \ldots\} ;
\end{array}
$$

and similarly for each number $=2$.

The properties of the numbers concerned indicated by $(b),(c)$ are fairly obvious. All the numbers being even, a divergence of digits in the frac. tions compared, when it occurs, will always come in an odd place, and so 
the fraction which has to be larger must have 1 there, while the other has 2 ; therefore if the first divergence between the numbers defining the fractions.is between numbers representing 2 's* the smaller number must come in the symbol for the larger fraction (this securing a digit 1 in that fraction -in an odd place - where the other still has 2); if the divergence is between numbers representing 1's, the reverse will be the case. Thus a proper arrangement in case $(b)$ will be $p<t$, or $p=t$ and $o>u$, or $p=t, o=u$ with a smaller number preceding $o$, in the direct sequence of numbers as given in (1) above, than that which follows $u$, and so on ; while in case (c) we must have $p>t$, or $p=t$ and $o<u$, etc. It will ensure that every $\lambda<3$, if the conditions just specified hold for every 2 flanked by unequal numbers, throughout the entire sequence of numbers, it being understood that the numbers are all even, that one of every two consecutives is 2 and that the flanking numbers, when unequal, differ by 2 .

5. These laws, however, may be simplified when we have considered the possibilities of repeated doublets, $2=r=s=\ldots+$ Suppose that at any point $m$ consecutive numbers each $=2$, where $m>1$; then, by the alternatives in (3) of last section, 4 must stand on each side of this sequence of 2's, and by (2) the next number must again be 2 . Suppose that here there are $n$ 2's followed again by 4 , so that we have in succession $\ddagger$ $\ldots 4,2,2,2, \ldots$ ( $m$ times) $4,2,2, \ldots$ ( $n$ times) $, 4, \ldots$ Identifying the last 2 of the $m$-set with $r$ in the conditions of last section, $3(b)$ applies; and identifying the first 2 of the $n$-set with $r$ (supposing $n>1$ ) 3 (c) applies, giving respectively

$$
\begin{aligned}
& \{2|2| 2 \ldots(m-2 \text { times }) \mid 4 \ldots\}>\{2|2| 2 \ldots(n \text { times }), 4 \ldots\}, \\
& \{2|2| 2 \ldots(n-2 \text { times }) \mid 4 \ldots\}>\{2|2| 2 \ldots(m \text { times }), 4 \ldots\} .
\end{aligned}
$$

Exactly as in the preceding paragraph, if $m-2<n$, (i) implies that $m$ is odd-the 4 in the first bracket must represent 1's not 2's. So if $n-2<m$, (ii) implies that $n$ is odd. $m-2<n$ and $n-2<m$ may both be true: then $m=n$, since both must be odd. Suppose, however, that not only $m-2<n$ but $m<n-2$; then, by (i), $m$ should be odd, and by (ii) $m$ should be even, which is impossible. Similarly $n<m-2$ is

* I.e. representing 2's in the fractions compared in $(b),(c)$, not necessarily in the original fraction owing to transformations. See the end of $\S 3$.

$\dagger$ This is a question not definitely considered by Markoff.

$\ddagger$ It should hardly be necessary to emphasise that these 2's are not the digits of the continued fraction, but the numbers of successive 1's and 2's of which these digits consist. 
impossible. We may, however, have $m=n-2$ (and so $m-2<n$ ) if $m$ be odd, with a further condition, or $n=m-2$ and odd, with a further condition. Since either $m-2<n$ or $n-2<m$ must hold for any pair of numbers, these are the only possible cases, except that of $n=1$, which was put aside to start with. In that case the condition (ii) of this paragraph disappears [since $3(a)$ of $\$ 4$ holds good, if we take the isolated 2 for $r]$, and (i) becomes $\{2|2| 2 \ldots(m-2$ times $) \mid 4 \ldots\}>\{2 \mid 4 \ldots\},(n$ being by supposition $>1$ ); and this cannot hold unless $m-2=1$, $m=3$; since $\{2 \mid 2 \ldots\}$ and $\{4 \mid \ldots\}$ (the fractions which arise when $m-2>1$ or $=0$ ), are each less than $\{2 \mid 4 \ldots\}$. In every case then we have proved that $m$ and $n$ must both be odd. Since we saw before that one (at least) of every two consecutive numbers must be 2 , and now that the number of successive 2's must always be odd, it follows that every alternate place throughout the whole sequence must be occupied by 2 (though 2 may appear in other places likewise). Thus either the 1's or the 2's which constitute the digits of the continued fraction must occur in doublets only, from and after some fixed point (though there may also be doublets of the other).* We may therefore fix our attention exclusively' on the sequence of alternate numbers, giving the numbers of intermediate 2's or 1's, as the case may be ; and the properties enunciated at the end of $\S 4$ are much simplified. In the notation of that section we have, say, $p=r=t=\ldots=2$, and any possible divergences between the numbers of the ascending and descending sequences which define the fractions compared in $3(b),(c)$ will be between those which represent 1 's there. $\dagger$ Therefore in accordance with the rules of that section, the larger of the two first diverging numbers must always come in connection with the larger fraction of the two compared. Using $\ldots 2 a, 2 b, 2 c, \ldots$ to represent the alternate numbers, so that (understanding that the others are all 2's and that $\ldots a, b, c \ldots$ denote integers) law (1) and law (2) of $\$ 4$ are necessarily satisfied, we have the following rules for any two successive numbers $d, e$, identifying $2 d, 2 e$ with $q$, $s$ of that section :-

(I) Either $d=e$, or $e=d+1$, or $d=e+1$.

(II) If $d, e$ are unequal, the sequences $c, b, a, \ldots ; f, g, h, \ldots$, formed by taking the numbers backwards from $d$ and forwards from $e$, must, when they first diverge, have the larger number in the sequence which starts from the smaller of the two $d$, $e$-that being the sequence corresponding to the fraction which has to be the larger in accordance with (3), (b), (c).

* Markoff only shows explicitly the case of the 2's occurring in doublets, though the possible vanishing of certain numbers which he uses involves the other case.

$\dagger$ Not necessarily representing 1 's in the original fraction, as noted in $\$ 4$. 
E.g. if $e=d+1$, either $f<c$, or $f=c, g<b$ or $f=c, g=b, h<a$, and so on; while if $d=e+1$ we have $c<f$, etc. Conversely, if (I), (II) are satisfied for each successive pair of numbers, all the requirements for $\lambda<3$ are fulfilled.

6. If we consider a little further the implicutions of the above laws, remarkable consequences follow. To begin with, the numbers in the sequence, even when not all equal, will be very closely restricted. Suppose that, in the sequence $\ldots a, b, c, \ldots, c$ differs from $b$, but is the first of $h$ numbers each equal to $c$, followed by a different number $c^{\prime}$, where $h>1$. By $\S 5$ (I) $c^{\prime}=c \pm 1$ : suppose $c^{\prime}=c+1$. Then we have $c, c+1$ preceded by $c$ (in fact by $h-1 c$ 's) and therefore by $\$ 5$ (II) followed by a number $=c$ or less. By $\S 5$ (I), again, the number following $c+1$ cannot be less than $c$ : it must therefore $=c$, the first (suppose) of $k c$ 's. We thus have (a) $c, c+1$ (as indicated by the ordinates at $A, B$ in the graph below) preceded by $h-1 c$ 's and followed by $k c$ 's, and $(\beta) c+1, c$ (as at $B, C$ in the figure) preceded by $h c$ 's and followed by $k-1 c$ 's (Fig. 1). * From (a) by

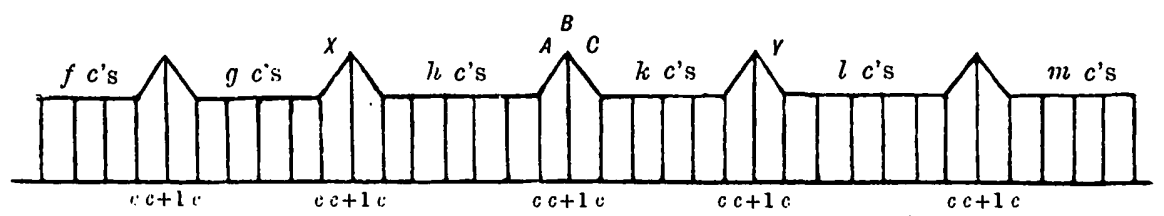

Fig. 1.

$\$ 5$ (II), if $k<h-1$, the (new) number following the $k c$ 's should be $<c$ (since the $k$-th number before $A$, the smaller of the two values $A, B$, is $c$ ); while by $(\beta)$ (from a like consideration of the numbers before and after $B C$ ) it should be greater, since $a$ fortiori $k-1<h$. Thus we cannot have $k<h-1$; nor (similarly) $h<k-1$ : i.e. $k$ must $=h-1$ or $h$ or $h+1$.

In the first place suppose that $k=h-1$ or $h$. Then $k-1<h$; and so, recurring to the sequences preceding and following $B C$, the number following the $k$-th $c$ (which by supposition is not $c$ ) must be $c+1$ by $\$ 5$ (II). If, of the alternatives now being considered, $k=h$, and therefore by hypothesis greater than unity, this $c+1$ must be followed by $c$, just as $c+1$ at $B$ involved $c$ at $C$; and similarly $c+1$ and then $c$ will precede the $h c$ 's, since $h-1<k$. If, however, $k=h-1$, it will still follow, from

* The object of the graph is not merely to make clearer to the eye the ups and downs of the sequence of numbers, but also to distinguish by letters $A, B, \ldots$ certain of these numbers from other equal numbers.

SER. 2. vol. 20 . No. 1392 . 
(a), that the $h-1 c$ 's before $A$ must be preceded by $c+1$, inasmuch as the $k c$ 's are followed by $c+1$ (see above), so that by $\S 5$ (II) the term cannot be $<c+1$, while by $\S 5$ (I) it cannot be greater; and again this $c+1$ must be preceded by $c$, as before. Therefore (again recurring to the sequences on eacb side of $A B$ ) even if $k=1$, so that the original argument for: $c+1$ at $B$ follonoed by $c$ at $C$ which was based on $h>1$, does not apply, the $c+1$ which follows the $k c$ 's must be followed by $c$ (as at $Y$ ) because the $c+1$ which precedes the $h-1 c$ 's is preceded by $c$ (at $X$ ), $k$ being $=h-1$; for by $\S 5$ (II) that at $Y$ cannot be greater than that at $X$, while by $\S 5$ (I) it cannot differ by more than a unit from $c+1$, which immediately precedes.

So far as noted, then, the sequences preceding and following $A B$, i.e. those back to $X$ and on to $Y$, inclusive, in the graph, Fig. 1, will agree in the case of $k=h-1$, as there shown. Suppose the $c$ at $X$ is the last of $g c$ 's and that at $y$ the first of $l c$ 's, which $g$ and $l c$ 's, again, by a repetition or extension* of the preceding arguments will be preceded and followed by $c+1, c$; and so on, indefinitely (so that the numbers throughout will be limited to the values $c$ and $c+1$ ). Then in order that, in the sequences following $c+1$ at $B$ and preceding $c$ at $A$, the larger number, when they diverge, may occur in the sequence starting from the lower, we must have $g<l$, if $g, l$ are unequal ; and then $c+1$ before the $g c$ 's will answer to $c$ in the other sequence, which is right; or, if $g=l$, then $f<m$, supposing $c+1$ and then $f c$ 's precede and $c+1$ and then $m c$ 's follow; or else $g=l$, $f=m$, with a like further condition; and so on. Similarly in the case of $h=k-1$, which was left aside, we should have $g>l$; or $g=l, f>m$; or $g=l, f=n$, with a like further condition; and so on. The result is that the whole sequence of numbers $\ldots a, b, c, \ldots$, restricted as we have

- An extension of the argument will be required if " singlets" are repeated. Suppose $h=2, k=1, l=1$, as in Fig. 2 ; the single $c$ (at $Y$ ) given by $l=1$, being necessarily followed by $c+1$ as before (because $c+1$ and then $c$ precede it). Then we must recur to the sequences preceding and following $A B$ to show that $c$ at $X$ must be preceded by $c+1$, answering to $c+1$ in the following sequence, so that $g=1$. Then $c+1, c$ immediately following $X$ will be the starting point of sequences showing that again $c$ must precede; and then again the sequences based on $c, c+1$ at $A, B$ show that $c$ must follow the $c+1$ after $Y$, the terms being determined in the order shown by (1), (2), (3), (4) in Fig. 2 ; and so on.

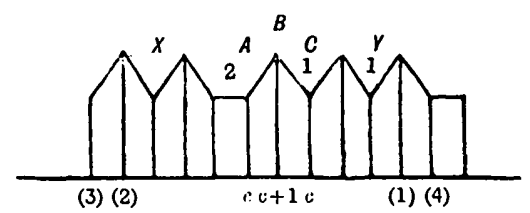

FIG. 2. 
seen to sets of $c$ 's separated by single $(c+1)$ 's, must be such that $\ldots f, g, h, k, l, m, \ldots$ the numbers of c's in the successive sets, obey precisely the same laws as those of the sequence which they thus define, namely, the laws $§ 5$ (I) and (II) which we have been using throughout the present section. This is all on the supposition that at least two consecutive $c$ 's occur somewhere and that the next number $c^{\prime}=c+1$. If $c^{\prime}=c-1$, we have, by precisely similar arguments, single $(c-1)$ 's separating groups of $\ldots f, g, h, \ldots, c$ 's, as indicated in Fig. 3, where $\ldots f, g, h, \ldots$ obey precisely the same laws as before.

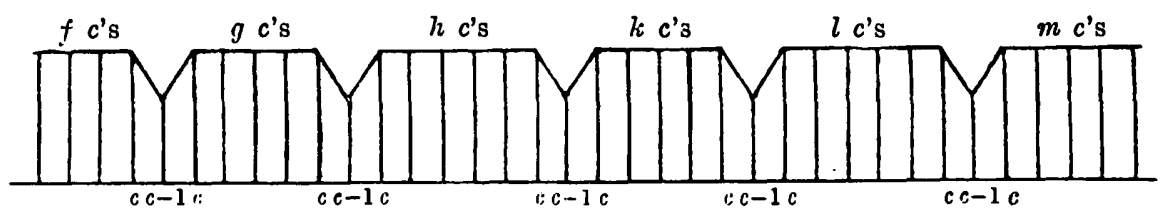

FIG. 3.

We may, however, have single c's alternating throughout with single $(c+1)$ 's, which we may consider a special case of either of the above with $f=g=\ldots=1$ (Fig. 4); or the still more rudimentary case where all the numbers are $c$ 's (Fig. 5) :-

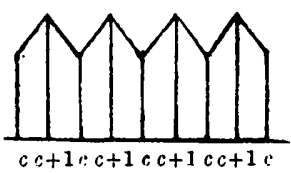

Fig. 4.

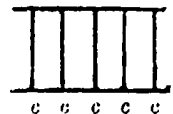

Fig. 5.

But the general result is that the primary sequence $\ldots a, b, c, \ldots$ consists of at most two different numbers, one of which, occurring singly, differs from the other by unity, while the occurrence of the other in sets of $\ldots f, g, h, \ldots$ together is "regulated" by the " secondary" sequence of the numbers $\ldots f, g, h, \ldots$, obeying precisely the same laws as the primary; also, since it obeys the laws $\$ 5$ (I) and (II) it must obey the further laws deduced from them in this section and be "regulated" by a like sequence $\ldots x, y, z, \ldots$, say, and so on; except that we can go no further when we reach a sequence of equal terms. Conversely, if the "regulating" sequence obeys the laws, this will hold also for the sequence which it regulates.

7. So far the work is on parallel lines to that of Markoff. Though his results are less symmetrically formulated; the laws of a sequence of 
numbers defining in the way described a sequence of "digits" 1,2 for which every $\lambda \leqslant 3$, where $\lambda$ is of the form

$$
a_{n+1}+\frac{1}{a_{n+2}}+\frac{1}{a_{n+3}}+\cdots+\frac{1}{a_{n}}+\frac{1}{a_{n-1}}+\cdots,
$$

the $a$ 's denoting the digits in order, hold for him and us alike. But for us the successive $a$ 's are the digits (or the digits from and after some fixed point) of the continued fraction for $\theta$, and therefore unlimited on one side only; and this has to be borne in mind in making our application of the results ; whereas for Markoff (as before pointed out) the sequence of $a$ 's is unlimited in both directions.

His application to the theory of the (numerical) minima of a set of equivalent quadratic forms such as $a x^{2}+2 b x y+c y^{2}$, where $a, b, c$ are given numbers, is briefly as follows. [It is supposed that $x, y$ are to receive integral values not both zero.] By linear transformations such a form can always be "reduced" to one

$$
a_{r} x_{r}^{\prime \prime}+2 b_{r} x_{r} y_{r}+c_{r} y_{r .}^{\prime}
$$

whose roots (i.e. the values of $x_{r} / y_{r}$ which make it vanish) are one positive and grenter than unity $=\xi_{r}$, say, and the other negative numerically less than unity $=-1 / \eta_{r}$. Supposing that

$$
\xi_{r}=a_{r}+\frac{1}{\alpha_{r+1}}+\cdots, \quad \eta_{r}=a_{r-1}+\frac{1}{a_{r-2}+}+\cdots
$$

(neitber terminating), the transformation

$$
x_{r}=a_{r} x_{r+1}+y_{r+1}, \quad y_{r}=x_{r+1}
$$

will give a new form of which the roots are

$$
a_{r+1}+\frac{1}{a_{r+2}+}+\cdots, \quad-\frac{1}{a_{r}}+\frac{1}{a_{r-1}}+\cdots ;
$$

and similarly through a whole series of transformations. Thus the $\lambda$ 's come in as the differences between the roots of a succession of equivalent reduced forms, got by using such transformations backwards and forwards; the $a$ 's here answering to the $a$ 's in the preceding paragraphs.

Unless all the $a$ 's are 1's we may take for our standard form, of this equivalent set, one $a x^{2}+2 b x y+c y^{2}$ for which the root $\xi>2$. For this, when $y=0$, the minimum, got by putting $x=1$, has the numerical value $|a|$, and since the form $\equiv a y^{2}(x / y-\xi)(x / y+1, \eta)$, it can be shown tbat, when $y$ is different from 0 , it can have no lower value than $|a|$ unless, supposing $x / y$ positive,$|x / y-\xi|<1 / 2 y^{2}$ and then $x / y$ must be a convergent to $\xi$, siny the $n$-th ; and the formulæ of transformation will show that the result of substituting such values of $x, y$ in the standard form is the same as the result of substituting 1,0 for the variables in the $n$-th form from the standard one, i.e. it is the $a$ of that form. Similarly, if $x / y$ is negative, $-x / y$ (if $x, y$ give a lower value) must be a convergent to $1 / \eta$, and we have a value which is the $a$ of some preceding form. Thus the minimum value required is that of the numerically least of the $a$ 's. But the difference of the roots of any form, which we have seen is one of the succession of $\lambda$ 's, is equal to $2 \sqrt{ } D /|a|$ where $a$ is the coefficient of $x^{2}$ in that form, $D$ being the determinant of the whole set of forms. It follows that the least value of $|a|$ is the same thing as the least value of $2 、 D / \lambda$, which is therefore the minimum required. In particular, if $\lambda<3$ throughout, the minimum is greater than $\frac{2}{3}, D$, and this is a critical value. If the coefficients $a, b, c$ are rational, the a's must recur. [The case of rational roots nced not be considered, as the form then has zero for its minimum.] 
Our concern is with the fact that so long as the successive $\lambda$ 's belonging to the continued fraction for $\theta$ are less than 3 , there can be no approximation $x / y$ to $\theta$ among the convergents to $\theta$ (where alone it could be found) such that $|x / y-\theta|<1 / 3 y^{2}$; and these $\lambda$ 's, like Markoff's, will be less than 3, provided the digits for $\theta$ consist of 1 's and 2's obeying the laws formulated in the preceding sections. But as the digits start from a fixed point the numbers which define them are also terminated in one direction. Now the final law of the numbers $\ldots a, b, c, \ldots$ which we reached in $\S 5$, is that the sequences taken onwards from the second and backwards from the first of two unequal consecutive terms must, when they diverge, diverge in a particular manner, but (though certain consequences have been further developed in $\$ 6$ ) the possibility of continual agreement without divergence has not yet been faced, and this introduces quite new considerations. In the sequences belonging to Markoff's problem, this possibility is of no great moment. Continued agreement, with him, is agreement to infinity, and that merely means that the corresponding $\lambda$, instead of being definitely less than 3 , takes the limiting value 3 ; a contingency impossible in our case (with $n$ finite) since our $\lambda$ is the sum of a terminating and an unending fraction. For us continual agreement without divergence can only mean agreement until the backward-reaching sequence terminates with the initial term of the whole series of numbers, and then the result $\lambda<3$, though not contradicted, is not guaranteed. Further examination is necessary to see whether after all $\lambda$ may not then be $>3$, in any given case.

We have then two or three possibilities to consider. Suppose, in the first place, that the proper divergences do always show themselves and always within a finite number of terms, a number $<N$, say, where $N$ is finite. Here Markoff's sequences (except that they have no beginning) are on the same footing as ours, and his digits, starting from some arbitrary point, would equally serve our purpose. Forming the successive derived sequences, as explained in the last section, since each is more "condensed" than the preceding, we shall at length reach a sequence where the "range of fulfilment" reduces to a single term, i.e. a sequence of equal numbers. This monotonous repetition involves a corresponding repetition of more extended range in the original sequence, and therefore in the continued fraction for $\theta$, so that $\theta$ must equal a quadratic surd. In such a case $\lambda$ will be always less than 3 by a certain finite amount at least (possibly very small) depending on the value of $N$. Conversely, any quadratic surd for which $\lambda$ beyond a certain point is always definitely less than 3 must depend on a sequence such as that described above, where the laws work 
themselves out within a finite range and lead ultimately to a sequence of equal terms. Further, since there will be an infinite number of sequences $\ldots a, b, c, \ldots$ of this character, there will be an infinite number of quadratic surds $\theta$ (though of narrowly restricted types) for which there are no approximations $x / y$ (or only a finite number) such that

$$
|x / y-\theta|<1 / 3 y^{2} \text {. }
$$

But suppose, on the other hand, that the digits are governed by a sequence of numbers for which, although such divergences as there are between the sequences which have to be compared are always in the right direction, instances of complete agreement continually crop up however far we go. In such a case everything may depend on the way in which the digits start. Even if they may be said to be governed from. the outset by the sequence of numbers, in the way supposed, it still has to be decided whether a doublet of the one digit, or, say, $2 a$ of the other digit (taking $a$ to be the initial number of the sequence) is to come first, and also which of these are 1's and which 2's. And if, as has been con. templated, there are digits preceding those thus determined, everything will depend on the arrangement of such initial digits, which may affect the question of $\lambda>$ or $<3$ at any distance ahead, when the sequence $a, b, c, \ldots$ is of the critical nature supposed. One way of forming a sequence which is certainly not of the character considered in the last paragraph is by taking for the primary sequence a set of numbers so adjusted that the primary is identical with the secondary. Then so far as the sequence obeys the laws at the start, it will do so throughout, the terms which follow being regulated by initial terms which do, and so on. indefinitely. Since, however, all the successive derived sequences will be identical, we shall never reach a sequence of equal numbers. Therefore (1) the corresponding fraction will not recur, (2) the laws will take longer and longer to work themselves out as we proceed. Such a case is indicated in Fig. 6, where it will be seen that the primary sequence $1212112 \ldots$ is identical with the secondary, determined by the numbers of 1 's in the successive sets of 1's in the primary (if in our reckoning of sets we ignore the initial 1 and begin with the first which has 2's on each side of it). Thus the secondary sequence begins with 1 (answering to the singlet in question) and this is followed by 2 , answering to the first doublet of 1 's, and so on. It will be found, however, that the laws $\$ 5$ (I), (II) are obeyed throughout only in the sense that divergences never occur in the wrong direction, and however far we go we have pair' of consecutive unequal terms such that the sequences which precede and follow agree without diverging until the backward one terminates; so that the case is such as was proposed for 
consideration at the beginning of this paragraph. Thus 21 at $H K$ are

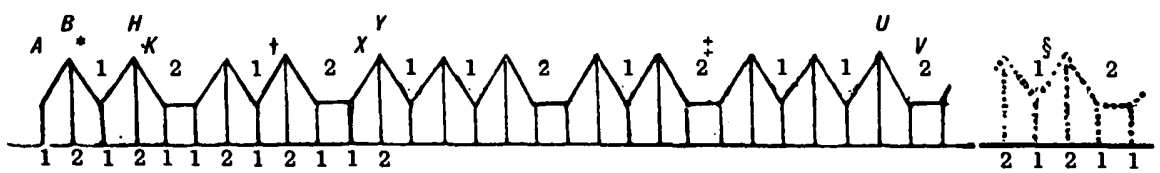

Fra. 6.

preceded by 121 and followed by $121 \ldots$, and similarly 12 at $X Y$ are preceded by 1212112121 (there terminating) and followed by the same numbers (with others beyond); and so continually at successive stages. This agreement is an index to a like agreement in the digits, and everything may therefore depend on how the initial digits are arranged. To examine how this works in detail, suppose that the doubles of the numbers of the sequence, viz. $2,4,2,4,2,2,4, \ldots$ indicate numbers of 2's separated by doublets of 1 's,* the alternation starting with 11 ; and that (if necessary) these again are preceded by other digits $\alpha, \beta, \gamma, \delta, \ldots$, so that the fraction written at length is

$$
\cdots \frac{1}{\delta}+\frac{1}{\gamma}+\frac{1}{\beta}+\frac{1}{\alpha}+\frac{1}{1}+\frac{1}{1}+\frac{1}{2}+\frac{1}{2}+\frac{1}{1}+\frac{1}{1}+\frac{1}{2}+\underset{B}{\frac{1}{2}+\frac{1}{2}+\frac{1}{2}+\frac{1}{1}+\cdots,}
$$

where the two 2's preceding $A$ and the four 2's divided into pairs at $B$ answer to the initial 12 of the number sequence marked $A B$ in Fig. 6. Then the condition to secure $\lambda<3$ for the first 2 of the $B$ group, which is critical owing to the agreement of digits before $A$ and after $B$, will be $[\$ 3(1)]$

$$
(2,2,1,1, a, \beta, \gamma, \delta, \ldots)<(2,2,1,1,2,2,1,1,2,2, \ldots),
$$

i.e.

$$
(a, \beta, \gamma, \delta, \ldots)<(2,2,1,1,2,2, \ldots) .
$$

Again, the second 21 of the sequence, marked $H K$ in the figure, is the next critical point, the numbers which follow up to the point marked + agreeing, as we have seen, with the 121 which precede; and so we have the condition [§ 3 (2), first form]

$$
\begin{aligned}
(2,2, \ldots, 1,1, \alpha, \beta, \gamma, \delta, \ldots) & >(2,2, \ldots, 1,1,2,2,2,2,1,1, \ldots), \\
\text { i.e. } \quad(\alpha, \beta, \gamma, \delta, \ldots) & >(2,2,2,2,1,1, \ldots) ;
\end{aligned}
$$

* It will be remembered that the doubles of the sequence numbers may be interpreted as giving either numbers of 1's separated by pairs of 2's, or numbers of 2's separated by pairs of 1 's; but the available initial digits might not correspond in the two cases if they involved other numbers besides 1 's and 2's. 
$\alpha, \beta, \gamma, \delta, \ldots$ coming now into comparison with the digits detined by the numbers 21 which follow $t$ in the sequence. Both conditions are satisfied by taking $a=2, \beta=2, \gamma=1, \delta=1$, or $a=2, \beta=2, \gamma=2, \delta=3$, and stopping there. Moreover we can show that if these two conditions are satisfied all subsequent conditions of a like nature will be satisfied, in virtue of the identity of the primary sequence with the secondary, each " critical" point corresponding to the preceding critical point in such a way that the conditions repeat themselves. Thus the 21 at $H K$, already examined, considered as belonging to the secondary sequence, answers to 2 ones followed by 1 one in the primary (in the neighbourhood of $X Y$ ), after which the grouping of 1's up to the point answering to $t$ will agree with that of those which precede; and this means that again we have $1 \mathrm{2}$ at $X Y$ with the preceding sequence of numbers taken right back to the beginning agreeing with the following sequence up to $\ddagger$, though there will be no other instance of this between $H K$ and $X Y$. Moreover as we have 2,1 beyond + (the point in the sequence following $H K$ which matches that where the back ward sequence preceding $H K$ ends), so in the sequence following $X Y$ we shall have 2 ones followed by 1 one at the corresponding stage (in the neighbourhood of \pm in the figure); and that means that we have 1,2 beyond the point $\ddagger$ where the correspondence between the sequence following and that preceding $X Y$ ends (owing to the termination of the latter), just as we have 12 immediately after $A B$. $\alpha, \beta, \gamma, \delta, \ldots$ therefore come into comparison as at first with the digits 22112222 ; and as the sequences now compared are based on 12 at $X Y$, we have the same condition (i) as was based on the consideration of the digits defined by 12 at $A B$.

Then, again, corresponding to 12 at $X Y$, we have 1 one followed by 2 ones in the neighbourhood of $U, V$; and so the sequence following 1 at $V$ will agree with the sequence preceding 2 at $U$, as far as it goes; and as we have $\ddagger$ followed by 12 , we shall have 1 one and then 2 ones at the corresponding stage, as indicated by the dotted lines (detached) in the figure, and so giving 21 beyond $\$$, the end of the new correspondence based on $U V$, exactly as we had 21 beyond $t$, in connection with the sequences based on $H K$, and leading as before to the condition (ii). And so continually the conditions for the critical stages will reduce to either (i) or (ii). Thus the continued fraction, with its initial digits determined as before specified, will have every $\lambda$, at least after the very first, less than 3.

We have thus proved that while there are an infinite number of quadratic surds $\theta$, for which there are no approximations such that

$$
|x| y-\theta \mid<1 / k y^{2},
$$


where $k=3$, there are also numbers which are not quadratic surds of which the same is true. It should be noticed, however, that while, in the case of the surd, every $\lambda$ is less than 3 by a certain definite amount (at least), $\lambda$ in the latter case, though always $<3$, will approach 3 indefinitely as we proceed; for, at the critical stages, the continued fractions whose difference measures the divergence of $\lambda$ from 3 will agree to an ever increasing number of digits. And this must hold in the case of any number not a quadratic surd of which the statement is true, whether of the kind considered in the present section, or corresponding to a sequence where the proper divergences do (at length) always show themselves. For if agreement always ceases within a limited number of digits in the fractions compared, and so within a limited number of terms in the number sequence, while each derived sequence is more "condensed" than the preceding, a sequence of equal numbers must at length be reached and the corresponding fraction will recur.

8. The above disposes of the case of $k=3$. We have to contrast with it the cases of $k>3$ and $k<3$. The former was dealt with at the beginning of the paper $(\$ 1)$, where it was shown that however slightly $k$ exceeds 3, there are an unenumerably infinite number of $\theta$ 's (including therefore transcendental values) for which there are no approximations (or a finite number) with $|x| y-\theta \mid<1 / k y^{2}$. The case of $k<3$ is covered in the main by what we saw incidentally in the last section-that for ? quadratic surd of the type specified $\lambda$ is throughout (or from some point) less than 3 by some definite amount (at least); and conversely that, if $\lambda$ is always less than 3 by a definite amount, however small, $\theta$ must be a quadratic surd. Thus where $\theta$ is such a surd, there will be at most only a finite number of approximations $x / y$ such that $|x| y-\theta \mid<1 / k: y^{2}$, where $k$ is some number $<3$, and for this to hold when $k<3, \theta$ must be such a surd. It is to be noted, however, that $k$ must be greater than $\sqrt{ } \sqrt{5}$, the value to which $\lambda$ tends when all the digits are 1's, or there will be no number $\theta$ for which the $\lambda$ 's beyond a certain point are all less than $k$. If, however, $3>k>\sqrt{ } 5$, there will always be quadratic surds to which the statement is applicable. Thus the proposed problem is completely solved. In the parallel case Markoff infers that there can be only a finite number of digit-sequences that will do for a given value of $k<3$, inasmuch as such a value implies a definite limit for $N(\S 7)$, and therefore a limited number of possibilities. He deduces that there can be only a limited number of quadratic forms with minima $\geqslant$ the corresponding fraction of $\sqrt{ } D$. We eannot, however, lay down that there will only be a finite number of $\theta$ 's for such a $k$, inasmuch as there may be any variety of digits preceding 
those which recur in the ways defined by such a sequence. But the recurring part of the continued fraction for $\theta$ will admit of only a finite variety of forms. The main conclusion however is that, if there are only a finite number of approximations $x / y$ such that $|x / y-\theta|<1 / k y^{2}, \theta$ must be a quadratic surd if $k<3$, but not otherwise.

9. Though it has nothing to do with our own problem, it may be of interest to notice, in conclusion, that in a way parallel to that of $\S 7$ we may construct a sequence such as Markoff uses, unlimited in either direction, where the primary sequence is identical with the secondary and therefore with all that follow, and which strictly obeys the sequence-laws (Fig. 7).

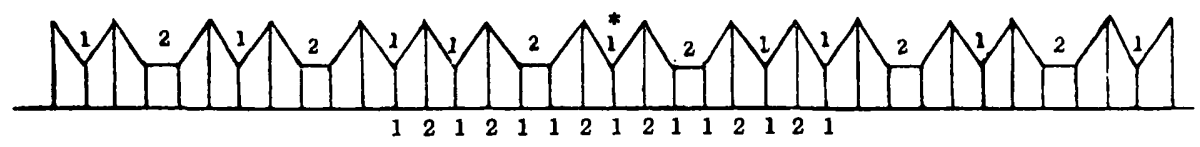

FIg. 7.

Here $\lambda$ will always be less than 3 , approaching however the limiting value 3 as we recede indefinitely from the central point of symmetry, shown by * in the figure. But the coefficients of corresponding quadratic forms would be irrational, not to say transcendental, functions. [See the paragraph in small print under $\S 7$.] 